\title{
AFRL-RI-RS-TR-2014-215
}

\section{ULTRASENSITIVE CHARGE DETECTION USING A CAVITY EMBEDDED SINGLE COOPER PAIR TRANSISTOR}

TRUSTEES OF DARTMOUTH COLLEGE

AUGUST 2014

FINAL TECHNICAL REPORT

\section{AIR FORCE RESEARCH LABORATORY INFORMATION DIRECTORATE}




\section{NOTICE AND SIGNATURE PAGE}

Using Government drawings, specifications, or other data included in this document for any purpose other than Government procurement does not in any way obligate the U.S. Government. The fact that the Government formulated or supplied the drawings, specifications, or other data does not license the holder or any other person or corporation; or convey any rights or permission to manufacture, use, or sell any patented invention that may relate to them.

This report was cleared for public release by the $88^{\text {th }}$ ABW, Wright-Patterson AFB Public Affairs Office and is available to the general public, including foreign nationals. Copies may be obtained from the Defense Technical Information Center (DTIC) (http://www.dtic.mil).

AFRL-RI-RS-TR-2014-215 HAS BEEN REVIEWED AND IS APPROVED FOR PUBLICATION IN ACCORDANCE WITH ASSIGNED DISTRIBUTION STATEMENT.

FOR THE DIRECTOR:

/ S /

PAUL M. ALSING

Work Unit Manager
/ S /

MARK H. LINDERMAN

Technical Advisor, Computing and Communications Division Information Directorate

This report is published in the interest of scientific and technical information exchange, and its publication does not constitute the Government's approval or disapproval of its ideas or findings. 


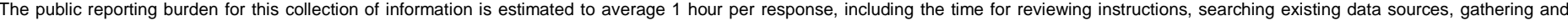

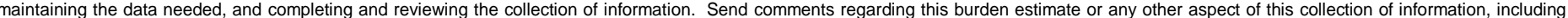

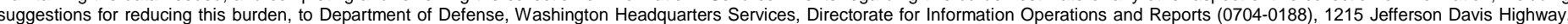

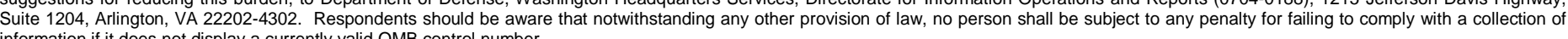

information if it does not display a currently valid OMB control number.
PLEASE DO NOT RETURN YOUR FORM TO THE ABOVE ADDRESS.

\begin{tabular}{|c|c|c|}
\hline $\begin{array}{c}\text { 1. REPORT DATE }(D D-M M-Y Y Y Y) \\
\text { AUGUST 2014 }\end{array}$ & 2. REPORT TYPE & 3. DATES COVERED (FrOm - To) \\
FINAL TECHNICAL REPORT & $2012-$ FEB 2014 \\
\hline
\end{tabular}

\section{TITLE AND SUBTITLE}

ULTRASENSITIVE CHARGE DETECTION USING A CAVITY

EMBEDDED SINGLE COOPER PAIR TRANSISTOR

\section{5a. CONTRACT NUMBER}

FA8750-12-2-0339

5b. GRANT NUMBER

N/A

5c. PROGRAM ELEMENT NUMBER

5d. PROJECT NUMBER

QEST

Alexander Rimberg

5e. TASK NUMBER

DA

5f. WORK UNIT NUMBER

RT

\begin{tabular}{l|ll}
\hline 7. PERFORMING ORGANIZATION NAME(S) AND ADDRESS(ES) & 8. & PERFORMING ORGANIZATION \\
Trustees of Dartmouth College & REPORT NUMBER
\end{tabular}

Office of Sponsored Projects

11 Rope Ferry Rd \#6210

Hanover, NH 03755

9. SPONSORING/MONITORING AGENCY NAME(S) AND ADDRESS(ES)

10. SPONSOR/MONITOR'S ACRONYM(S)

Air Force Research Laboratory/RITA

AFRL/RI

525 Brooks Road

11. SPONSOR/MONITOR'S REPORT NUMBER

Rome NY 13441-4505

AFRL-RI-RS-TR-2014-215

\section{DISTRIBUTION AVAILABILITY STATEMENT}

Approved for Public Release; Distribution Unlimited. PA\# 88ABW-2014-3781

Date Cleared: 14 AUG 14

\section{SUPPLEMENTARY NOTES}

\section{ABSTRACT}

We have investigated an alternate approach to standard charge sensing of quantum dots Dsbased on the radiofrequency single electron transistor (RF-SET) or quantum point contact (RF-QPC). Our approach should be more sensitive and less invasive than standard approaches, and allow higher device density. By embedding a

superconducting SET known as a Cooper pair transistor (CPT) in a superconducting microwave cavity, it is possible to use the charge sensitivity of the CPT's quantum inductance to dispersively tune the cavity resonant frequency. A carrier wave at the resonant frequency then experiences a charge-dependent phase shift. Charge detection is in turn achieved via interferometric measurement of the phase of a transmitted carrier wave. In this detection scheme the CPT is operated on its supercurrent branch and contributes no shot noise, allowing the usual shot-noise limit on charge sensitivity to be beaten. Furthermore, multiple CPTs could be embedded in the same cavity allowing at least four times the areal device density possible for the RF-SET. Finally, the detection process should be quantum limited, having the minimal effect on a measured QD allowed by quantum mechanics.

\section{SUBJECT TERMS}

Charge detection, Cooper pair transistor, quantum dot

\begin{tabular}{|c|c|c|c|c|c|}
\hline \multicolumn{3}{|c|}{ 16. SECURITY CLASSIFICATION OF: } & \multirow{2}{*}{$\begin{array}{l}\text { 17. LIMITATION OF } \\
\text { ABSTRACT } \\
\text { UU }\end{array}$} & \multirow{2}{*}{$\begin{array}{c}\text { 18. NUMBER } \\
\text { OF PAGES } \\
17\end{array}$} & \multirow{2}{*}{\begin{tabular}{|l|} 
19a. NAME OF RESPONSIBLE PERSON \\
PAUL M. ALSING \\
19b. TELEPHONE NUMBER (Include area code) \\
315-330-4960
\end{tabular}} \\
\hline $\begin{array}{c}\text { a. REPORT } \\
\text { U }\end{array}$ & $\begin{array}{c}\text { b. ABSTRACT } \\
\text { U }\end{array}$ & $\begin{array}{c}\text { c. THIS PAGE } \\
\text { U }\end{array}$ & & & \\
\hline
\end{tabular}




\section{TABLE OF CONTENTS}

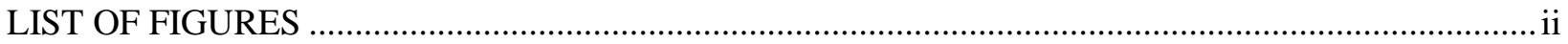

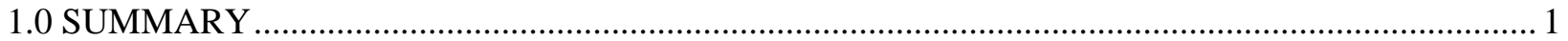

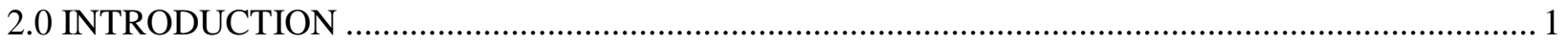

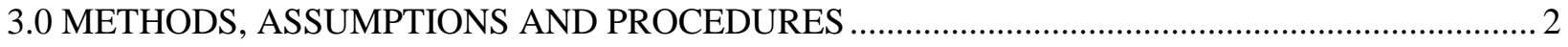

3.1 The cavity-embedded Cooper pair transistor .......................................................................... 2

3.2 Dispersive charge sensing using the

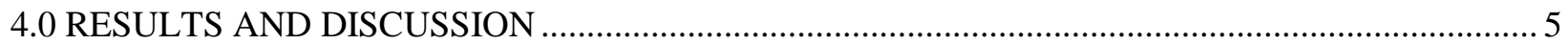

4.1 Development of superconducting microwave cavities.............................................................. 5

4.2 Installation of cryogenic microwave circuitry …................................................................. 7

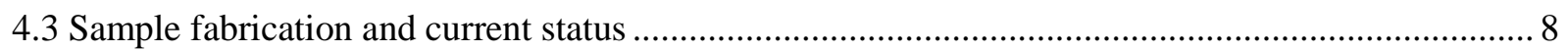

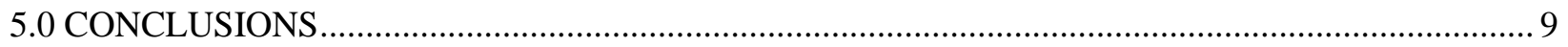

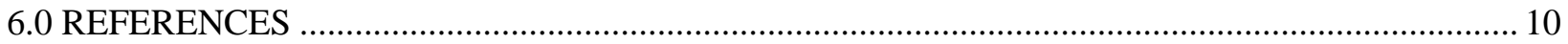

7.0 LIST OF SYMBOLS, ABBREVIATIONS, AND ACRONYMS …................................................ 12 


\section{LIST OF FIGURES}

Figure 1: Schematic of a cavity-embedded Cooper pair transistor ............................................................. 2

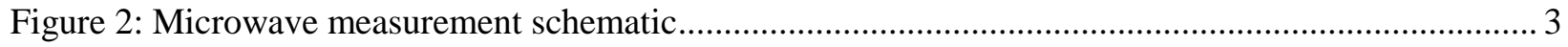

Figure 3: Optical micrographs of a quarter wave superconducting microwave cavity............................... 5

Figure 4: Spectrum of microwave power reflected from a quarter-wave superconducting cavity ............... 6

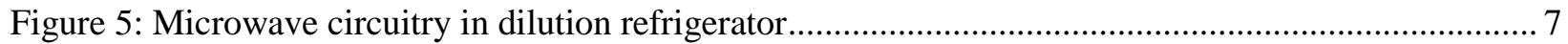

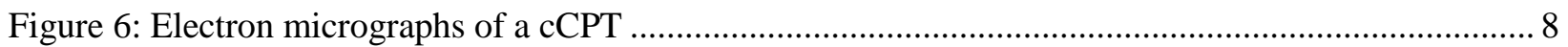




\subsection{SUMMARY}

We have investigated an alternate approach to standard charge sensing of quantum dots (QDs) on the radio-frequency single electron transistor (RF-SET) or quantum point contact (RF-QPC). Our approach should be more sensitive and less invasive than standard approaches, and allow higher device density. By embedding a superconducting SET known as a Cooper pair transistor (CPT) in a superconducting microwave cavity, it is possible to use the charge sensitivity of the CPT's quantum inductance to dispersively tune the cavity resonant frequency. A carrier wave at the resonant frequency then experiences a charge-dependent phase shift. Charge detection is in turn achieved via interferometric measurement of the phase of a reflected carrier wave. In this detection scheme the CPT is operated on its supercurrent branch and contributes no shot noise, allowing the usual shot-noise limit on charge sensitivity to be beaten. Furthermore, multiple CPTs could be embedded in the same cavity allowing at least four times the areal device density possible for the RF-SET. Finally, the detection process should be quantum limited, having the minimal effect on a measured QD allowed by quantum mechanics.

\subsection{INTRODUCTION}

At present, the charge detectors most frequently used for readout of QD-based spin qubits are the radio-frequency single-electron transistor (RF-SET) [Schoelkopf1998] and the closely related radio-frequency quantum point contact (RF-QPC) [Reilly2007]. Both operate on the same principle. The SET or QPC sensor is embedded in a matching network consisting of an inductor and an associated parasitic capacitance that serves to impedance match the sensor to standard $\mathbf{5 0} \Omega$ microwave electronics. The SET or QPC is also coupled to a nearby QD; when an electron tunnels on or off the QD, the sensor conductance changes, in turn changing the reflection coefficient of an applied carrier wave. Information regarding the QD charge state is therefore encoded in amplitude modulation of the reflected wave.

The RF-SET is necessarily a dissipative device, with a best sensitivity of roughly $\boldsymbol{\delta} \boldsymbol{q} \approx \mathbf{1} \times$ $\mathbf{1 0}^{-6} \boldsymbol{e} / \sqrt{\mathrm{Hz}}$ that is fundamentally limited by the shot noise of the current driven by the carrier wave. Furthermore, the fact that each RF-SET on a chip must have its own matching inductor (to minimize the parasitic capacitance) imposes a strict limit on the density of RF-SETs or RFQPCs on a chip to roughly one per $\mathbf{2} \mathrm{mm}^{2}$.

Here we have developed an alternate approach to charge sensing that should be more sensitive and less invasive, and have higher device density that is possible using the standard RF-SET configuration. By embedding a superconducting SET known as a Cooper pair transistor (CPT) in a superconducting microwave cavity, it is possible to use the charge sensitivity of the CPT's quantum inductance to dispersively tune the cavity resonant frequency. A carrier wave at the resonant frequency then experiences a charge-dependent phase shift. Charge detection is in turn achieved via interferometric measurement of the phase of a transmitted carrier wave. 
In this detection scheme the CPT is operated on its supercurrent branch and contributes no shot noise, allowing the usual shot-noise limit on charge sensitivity to be beaten. Furthermore, multiple CPTs could be embedded in the same cavity, allowing four or more charge detectors to be placed in a roughly $1.25 \times 2.5 \mathrm{~mm}^{2}=3.1 \mathrm{~mm}^{2}$ area, at least four times the areal density possible for the RF-SET. Finally, the detection process should be quantum limited, having the minimal effect on a measured QD allowed by quantum mechanics.

Our goal for this research has been to demonstrate that the cavity-embedded CPT (cCPT) can have a charge sensitivity as low as $\boldsymbol{\delta} \boldsymbol{q} \approx \mathbf{5} \times \mathbf{1 0}^{-\mathbf{8}} \boldsymbol{e} / \sqrt{\mathrm{Hz}}$, a factor of 40 better than the best value of $\boldsymbol{\delta} \boldsymbol{q} \approx 2 \times \mathbf{1 0}^{-\mathbf{6}} \boldsymbol{e} / \sqrt{\mathrm{Hz}}$ achieved in our laboratory for an RF-SET with an on-chip matching network. We have successfully installed required microwave components in a dilution refrigerator, designed and fabricated superconducting microwave cavities, and fabricated a cCPT suitable for measurement. Final measurements have unfortunately been hindered by a leak in the dilution refrigerator, and will be concluded after submission of this report.

\subsection{METHODS, ASSUMPTIONS, AND PROCEDURES}

\subsection{The cavity-embedded Cooper pair transistor}

In this research we have embedded CPTs in superconducting microwave cavities based on a modified form of the circuit QED (cQED) architecture [Wallraff2004, Blais2004], as illustrated schematically in Fig. 1. We refer to the CPT/cavity device as the cavity-embedded Cooper pair transistor or cCPT. Our goal is to use the gate-charge-tunable quantum inductance [Sillanpaa2004] of the CPT to induce a charge-sensitive dispersive shift in the resonant frequency of the cavity. A carrier wave reflected by the cavity will experience a charge-sensitive phase shift that can be detected using microwave interferometry [Blais2004, Regal2008].

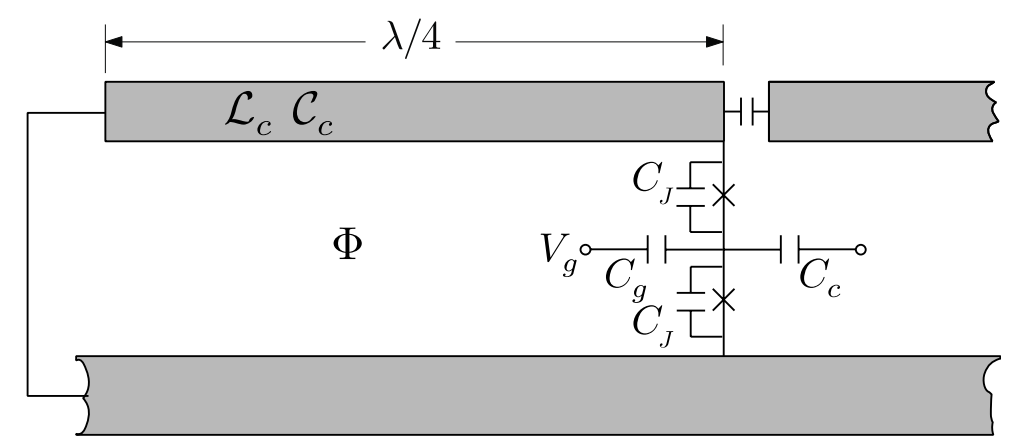

Figure 1. Schematic of a cavity-embedded Cooper pair transistor. The CPT is placed at the open end of a quarter-wavelength shorted superconducting microwave cavity. A gate voltage and external flux are used to control the CPT operating state. The cavity is capacitively coupled to a microwave feedline.

A CPT is added to the cavity at its voltage antinode such that it connects the central conductor to the ground plane. This connection differs from that recently used for cavity-embedded junctions that have been placed within the central conductor itself [Manucharyan2007] and allows the 
charge sensitive properties of the CPT to control the cavity's resonant frequency. Specifically, this is accomplished via the charge tunable quantum inductance $L_{\mathrm{CPT}}$ of the CPT associated with its supercurrent [Sillanpaa2004].

At the resonant frequency $\omega_{0}$ of the cavity, we can view the cavity as an effective parallel $L C R$ resonant circuit. The CPT is connected in parallel with the cavity circuit, and its quantum inductance $L_{\mathrm{CPT}}$ therefore appears in parallel with the effective cavity inductance $L$ giving rise to a strongly charge-sensitive cavity pull

$$
\left|\frac{\partial \omega_{0}}{\partial L_{\mathrm{CPT}}}\right| \approx \frac{1}{2} \frac{\omega_{0} L}{L_{\mathrm{CPT}}^{2}} .
$$

The notion of using the quantum inductance of the CPT for charge detection is not new. Both dissipative readout in low- $Q(Q \approx 20)$ resonant circuits [Sillanpaa2004] and dispersive readout in high- $Q(Q \approx 3000)$ resonators weakly coupled to a transmission line [Bell2011] have been explored experimentally. The combination of strong coupling and high $Q$ possible in the cCPT allows for the possibility of much better charge sensitivity than that achieved in that earlier work.

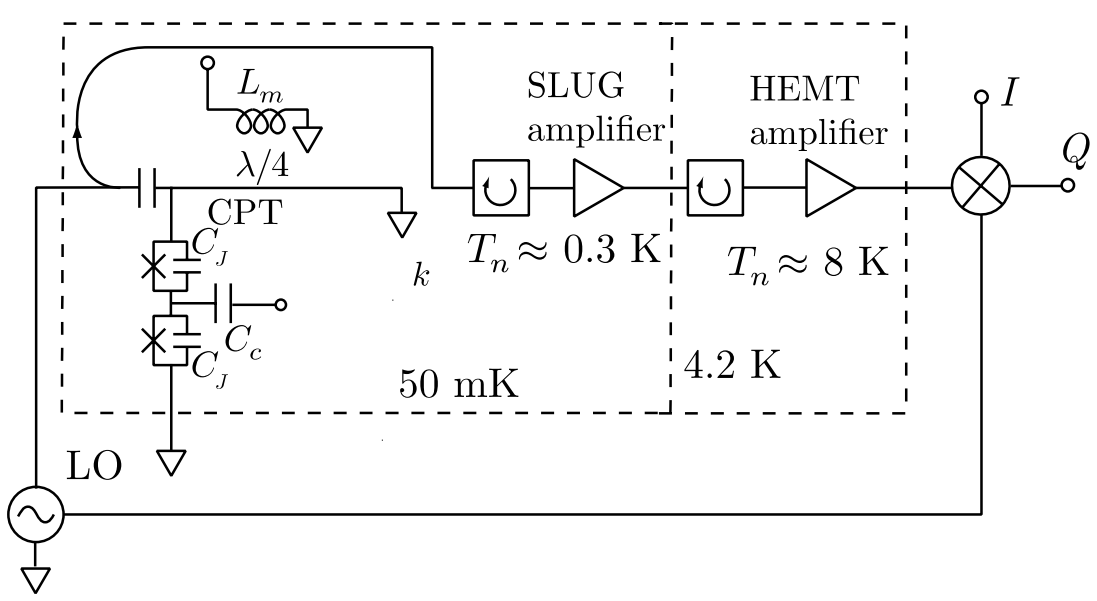

Figure 2. Microwave measurement schematic. The microwave signal reflected from the cCPT is amplified by a low-noise SLUG amplifier and a cryogenic HEMT amplifier before homodyne detection at room temperature.

While the charge dependence of CPT quantum inductance is well established, simultaneous control over both the low frequency and high frequency electrical environment of the CPT is needed to fully realize its potential. Low frequency control of the total current flowing through the CPT is needed to ensure that it is safely biased on its supercurrent branch. In our scheme, this will be accomplished by embedding the CPT in a shorted quarter-wave microwave cavity, as in Fig. 1. An externally applied magnetic flux will then be used to set the appropriate dc bias conditions. At the same time, embedding the CPT in a high-Q microwave resonator allows for high resolution dispersive readout. 


\subsection{Dispersive charge sensing using the CCPT}

Our dispersive readout scheme works as follows. An incident carrier wave at the resonant frequency of the CCPT is split into two parts: a wave that is reflected by the CCPT and a reference wave, as in Fig. 2. Because the CPT quantum inductance is gate charge dependent, the cCPT experiences a very strong charge sensitive cavity pull $\partial \omega_{0} / \partial n_{g}$ where $n_{g}=C_{g} V_{g} / e$ is the gate charge and $C_{g}$ and $V_{g}$ are the gate capacitance and voltage respectively. Using known experimental results [Sillanpaa:2005], we estimate that

$$
\left|\frac{\partial \omega_{0}}{\partial n_{g}}\right|=\left|\frac{\partial \omega_{0}}{\partial L_{\mathrm{CPT}}}\right| \frac{\partial L_{\mathrm{CPT}}}{\partial n_{g}} \approx 2 \pi \times 350 \mathrm{MHz} / \text { electron. }
$$

For a typical cavity $Q \approx 5000$ this corresponds to many linewidths $\kappa=\frac{\omega_{0}}{Q} \approx 2 \pi \times 1 \mathrm{MHz}$.

The wave reflected by the cCPT experiences a phase shift that, due to the cavity pull, is in turn strongly charge dependent. Measurement of the phase shift of the transmitted wave, which is achieved by mixing it with the reference wave, therefore gives information regarding the charge state of the CPT. We can estimate the expected spectral density of the resulting charge noise $S_{q q}$ using the expression [Teufel2009]

$$
S_{q q} \approx \frac{n_{\mathrm{add}}}{\bar{n}} \frac{\omega_{0}}{4 Q}\left[\frac{\partial \omega_{0}}{\partial n_{g}}\right]^{2}
$$

where $n_{\text {add }}$ is the number of noise photons added by subsequent amplifiers and $\bar{n}$ is the number of drive photons in the cavity. We use $\bar{n} \approx 500$ as a conservative estimate of the number of drive photons that can be used without exceeding the critical current of the CPT [Sillanpaa2005c].

We intend to amplify the transmitted wave using SLUG-based [Hover2012] and cryogenic HEMT amplifiers prior to mixing with the reference wave. The SLUG, or superconducting lowinductance undulating galvanometer, allows construction of amplifiers with a noise temperature of $1 \mathrm{~K}$. Such an amplifier has been provided by our collaborator Prof. Robert McDermott of the University of Wisconsin, Madison. Operating at $5.9 \mathrm{GHz}$, the SLUG will add approximately $n_{\text {add }} \approx 4$ noise photons per unit bandwidth to the measurement. Using the other parameters from above, and assuming a cavity $Q$ of 5000 , we obtain $S_{q q}=2.5 \times 10^{-15} e^{2} / \mathrm{Hz}$ or in terms of the more familiar charge sensitivity $\delta q=\sqrt{S_{q q}}$,

$$
\delta q \approx 5 \times 10^{-8} e / \sqrt{\mathrm{Hz}}
$$

This estimate is a factor of 40 better than that of an RF-SET with on-chip matching of $\boldsymbol{\delta} \boldsymbol{q} \approx \mathbf{2} \times$ $\mathbf{1 0}^{-6} \boldsymbol{e} / \sqrt{\mathrm{Hz}}$, which itself is on the order of the shot-noise limit and cannot be improved 
significantly [Xue2009]. Finally, we note that we have recently performed a more quantum mechanically exact calculation of the charge sensitivity of the cCPT, and found it to be in good agreement with the more empirical calculation above [Rimberg2013]. Clearly, the CCPT should be a far more sensitive electrometer that is physically possible for the RF-SET.

\subsection{RESULTS AND STATUS}

\subsection{Development of superconducting microwave cavities}

In order for the CCPT to work as designed, we require a high-Q superconducting cavity that can allow for highly coherent coupling between the cavity photons and the CPT supercurrent. In order to accomplish this, we turned to technologies developed in collaboration with the Simmonds group at NIST, Boulder. We desired Nb-based cavities for ease of initial testing, and superior behavior in a non-zero magnetic field. We also required that the cavities have a sloping sidewall, and Au contact pads at the planned CPT location, so that coherent flow of Cooper pairs could occur between the cavity conductor and the CPT.

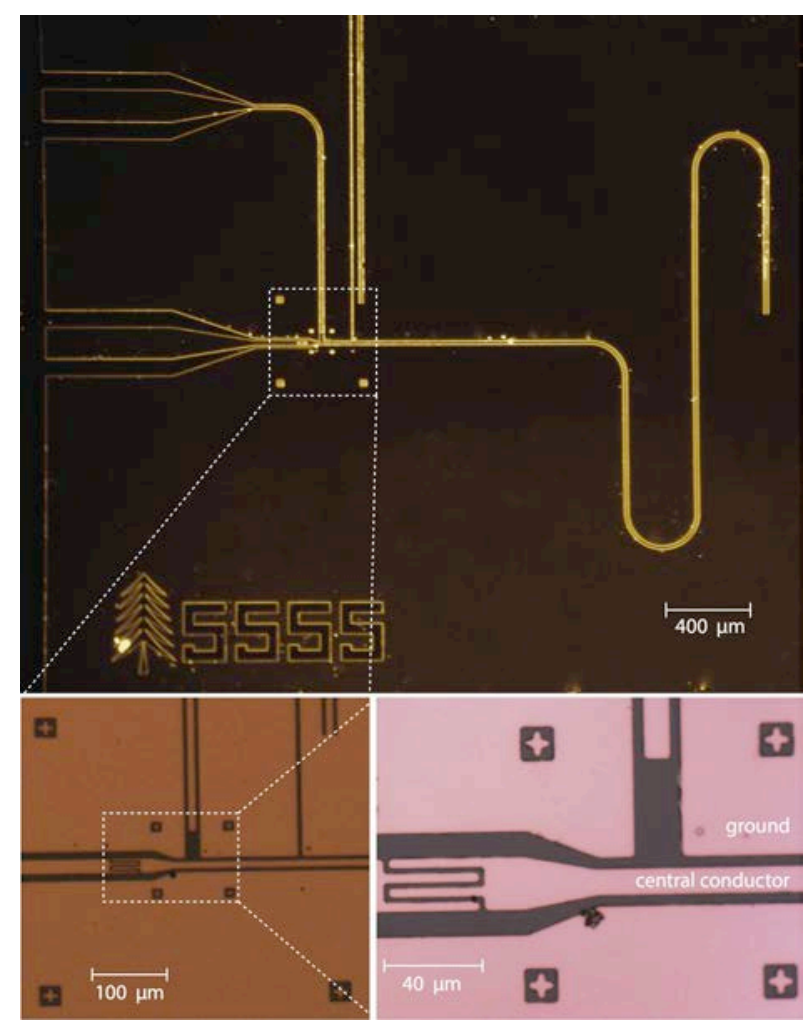

Figure 3. Optical micrographs of a quarter wave superconducting microwave cavity. Top: Overview of the entire cavity. Bottom left: Detail of the coupling capacitor, gate line for the CPT, and flux biasing line. Bottom left: Close-up view of the coupling capacitor and gate line. 
In our previous work with the Simmonds group, we had successfully used such techniques to produce dc-biased full-wave microwave cavities [Chen2011] that contained thin Au contact pads for good metallic contact. The Au pads were designed to be sufficiently thin that the proximity effect due to their contact with thicker layers of $\mathrm{Nb}$ (the cavity) and Al (the CPT) would drive them superconducting. Microwave and dc measurements of CPTs embedded in such cavities indicated that this scheme in fact worked quite well, as indicated by coherent transport in and photon emission from the embedded CPTs [Chen2013].

Here, rather than a full-wave cavity with dc bias lines we require a quarter-wave cavity with a flux bias line. Furthermore, we desired a larger cavity $Q$ of roughly 5,000, rather than the $Q=$ 3,500 we had chosen in our previous design. The most challenging aspect of the cavity redesign was to add a flux bias line that had minimal microwave coupling to the quarter-wave cavity. This was eventually accomplished by arranging the flux bias line at right angles to the cavity center conductor, while extending a portion of the center conductor/ground plane gap of the cavity parallel to the flux bias line. Simulations using the microwave design program Sonnet indicated that high-Q cavities with little cavity/flux bias line coupling could be achieved in this fashion.

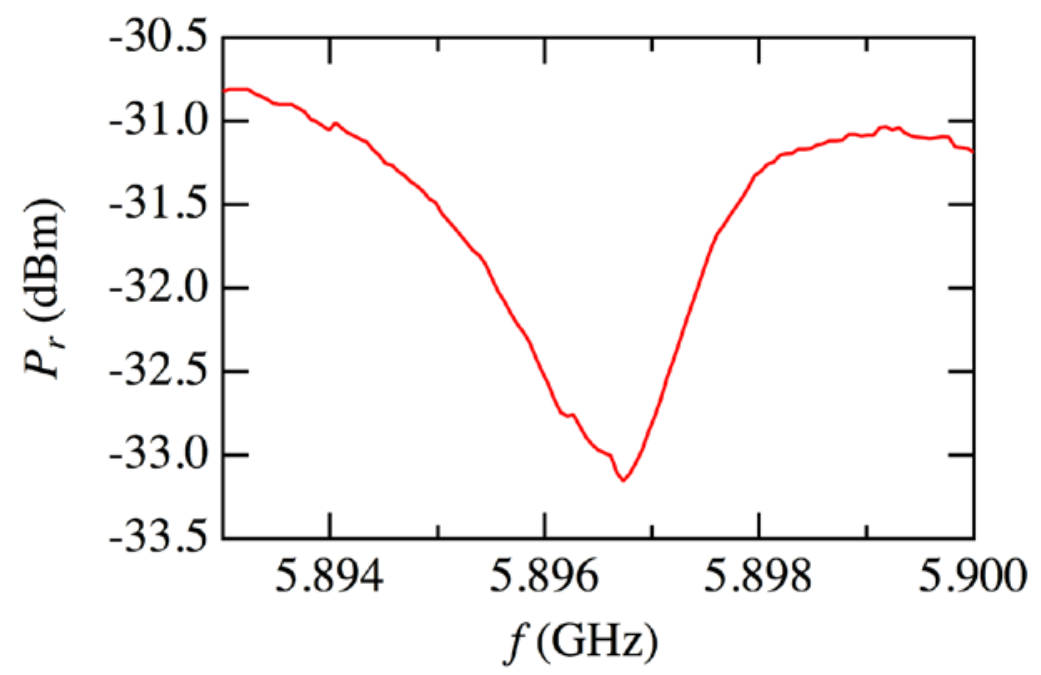

Figure 4. Spectrum of microwave power reflected from a quarter-wave superconducting cavity. The quality factor deduced from this spectrum is approximately 5,000.

We fabricated a set of resonators based on the above design principles in the laboratory of our collaborator, Prof. Robert McDermott at the University of Wisconsin, Madison. The resonators were produced by sputtering $100 \mathrm{~nm}$ of $\mathrm{Nb}$ and then using a $\mathrm{SF}_{6} / \mathrm{O}_{2}$ dry etch to remove the $\mathrm{Nb}$ in the gaps between the central conductor, gate line, and flux bias line on the one hand and the surrounding ground plane on the other. The $\mathrm{SF}_{6} / \mathrm{O}_{2}$ etch was verified to produce sloping side walls with a $45^{\circ}$ pitch. Ion milling was used to open vias through the native oxide of the $\mathrm{Nb}$, and $30 \mathrm{~nm}$ thick Ti/Au contact pads were deposited through the vias using e-beam evaporation. A typical resonator is shown in Fig. 3, along with detailed views of the cCPT location. 
The resulting resonators were tested at $4 \mathrm{~K}$ in our laboratory at Dartmouth; results are shown in Fig. 4. We found a clear resonance with a $Q$ of about 5,000 at a resonant frequency of roughly $5.9 \mathrm{GHz}$. There was also negligible coupling between the cavity and either the gate line or flux bias line, as desired. In contrast, however, the resonant frequency was roughly $9 \%$ higher than the design frequency of $5.5 \mathrm{GHz}$, possibly due to slight over-etching during removal of the $\mathrm{Nb}$. Still, the results were within the operating bandwidth of our microwave circuitry, allowing us to proceed with sample fabrication, to be discussed below.

\subsection{Installation of cryogenic microwave circuitry}

Since our most recent set of experiments involved transmission measurements at $5 \mathrm{GHz}$ and did not require use of a SLUG amplifier, significant effort was required to prepare our dilution refrigerator for reflection measurements using an additional amplifier.

Three major modifications were required. First, we installed a $5 \mathrm{GHz}$ directional coupler to allow conversion to reflection measurements. Second, we installed the SLUG amplifier itself, provided to us by the group of Prof. Robert McDermott at the University of Wisconsin, Madison. The SLUG had to be installed at the mixing chamber of the dilution refrigerator, since obtaining the best possible noise performance from the SLUG requires operation at the lowest possible temperature. The SLUG also required installation of two bias tees and resistive biasing networks for the flux bias and bias current required for its operation.

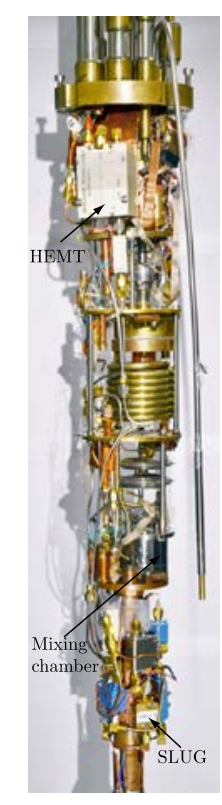

Figure 5. Microwave circuitry in dilution refrigerator. The SLUG and HEMT amplifiers are visible near the bottom and top of the image, respectively.

Finally, because the gain and noise of the SLUG are strongly dependent on its flux and current bias conditions, we installed two cryogenic microwave switches. These switches will allow us to bypass the SLUG while the cryostat is cold, allowing us to compare overall gain and noise of just the HEMT, and of the SLUG and HEMT in series. This capability will allow us to both verify 
correct operation of the SLUG and more accurately determine and characterize its optimal operating point. The various components of the completed cryogenic microwave circuit are shown in Fig. 5.

\subsection{Sample fabrication and current status}

Once work on the dilution refrigerator was completed, we began work on sample fabrication. Samples are produced by electron beam lithography and shadow evaporation of Al in a thermal evaporator. The Al-based cCPT and its leads needed to be aligned to prior fabrication steps with an accuracy of less than one micron.
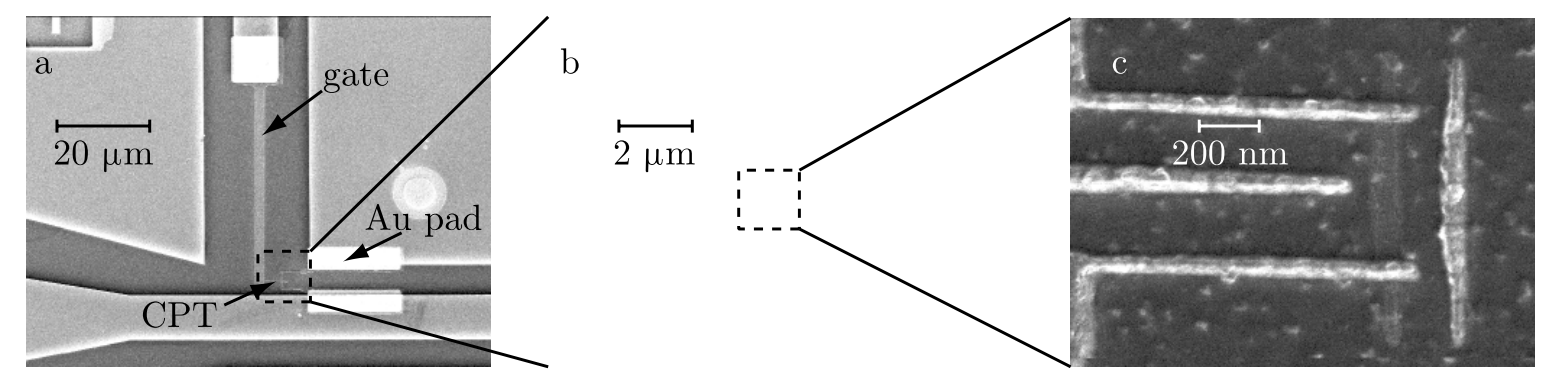

Figure 6. Electron micrographs of a cCPT. (a) Overview of the CPT location, showing the gate line and Au contact pads. (b) and (c) Successive close-up views of the CPT and its leads, and the CT itself.

Results from a typical fabrication sequence are shown in Fig. 6(a)-(c). An overview of the completed sample at the CPT location, including the gate line and Au contact pads, is shown in Fig. 6(a). Successive close up views of the cCPT are shown in Fig. 6(b) and (c). The substrate roughness in Fig. 6(c) is due to the ion milling step used to create the vias in the Nb oxide. Note that the actual CPT island is very faint; this is because the island is intentionally fabricated out of a very thin (7 nm) layer of $\mathrm{Al}$ to prevent trapping of quasiparticles on the island [Xue2009].

At present a sample similar to the one shown in Fig. 6 is in our dilution refrigerator awaiting measurement. Unfortunately, a cold leak in the inner vacuum can of the refrigerator has prevented us from performing anything more than very preliminary measurements showing that the SLUG amplifier is in fact operational. Once the location of the leak is determined and repairs performed, measurements of the $\mathrm{CCPT}$ performance will begin in earnest. 


\subsection{CONCLUSIONS}

The CCPT shows great promise for ultra-sensitive, quantum limited charge detection of such interesting systems as spin qubits and nanomechanical resonators. The cCPT promises to outperform the most commonly used fast mesoscopic charge sensors, namely the RF-SET and the RF-QPC, while potentially possessing a greater areal density. During the seventeen month period funded by this grant, we designed and fabricated superconducting quarter wave microwave cavities suitable for integration with a CPT. We modified an existing dilution refrigerator to allow microwave reflectometry measurements using an amplifier chain consisting of SLUG and HEMT amplifiers. We have successfully integrated a CPT with one of our superconducting cavities; the resulting sample is currently in our modified dilution refrigerator awaiting measurement. 


\subsection{REFERENCES}

[Bell2012] M. T. Bell, L. B. Ioffe, and M. E. Gershenson, "Microwave Spectroscopy of a Cooper-Pair Transistor Coupled to a Lumped-Element Resonator,” Phys. Rev. B 86, 144512 (2012).

[Blais2004] A. Blais, R.-S. Huang, A. Wallraff, S. M. Girvin, and R. J. Schoelkopf, “Cavity Quantum Electrodynamics for Superconducting Electrical Circuits: An Architecture for Quantum Computation,” Phys. Rev. A 69, 062320 (2004).

[Chen2011] F. Chen, A. J. Sirois, R. W. Simmonds, and A. J. Rimberg, "Introduction of a DC Bias into a High-Q Superconducting Microwave Cavity,” Appl. Phys. Lett. 98, 132509 (2011).

[Chen2013] Fei Chen, Juliang Li, A. D. Armour, E. Brahimi, Joel Stettenheim, A. J. Sirois, R. W. Simmonds, M. P. Blencowe and A. J. Rimberg, “A Single-Cooper-Pair Josephson Laser,” arXiv:1311.2042.

[Hover2012] D. Hover, Y. F. Chen, G. J. Ribeill, S. Zhu, S. Sendelbach, and R. McDermott, "Superconducting Low-Inductance Undulatory Galvanometer Microwave Amplifier," Appl. Phys. Lett. 100, 063503 (2012).

[Manchurayan2007] V. E. Manucharyan, E. Boaknin, M. Metcalfe, R. Vijay, I. Siddiqi, and M. Devoret, "Microwave Bifurcation of a Josephson Junction: Embedding-Circuit Requirements," Phys. Rev. B 76, 014524 (2007).

[Regal2008] C. A. Regal, J. D. Teufel, and K. W. Lehnert, "Measuring Nanomechanical Motion with a Microwave Cavity Interferometer,” Nature Phys. 4, 555 (2008).

[Reilly2007] D. J. Reilly, C. M. Marcus, M. P. Hanson, and A. C. Gossard, "Fast Single-Charge Sensing with an RF Quantum Point Contact,” Appl. Phys. Lett. 91, 162101 (2007).

[Rimberg2013] A. J. Rimberg, M. P. Blencowe, A. D. Armour and P. D. Nation "A CavityCooper Pair Transistor Scheme for Investigating Quantum Optomechanics in the Ultra-Strong Coupling Regime,” arXiv:1312.7521; to appear in New J. Phys.

[Schoelkopf1998] R. J. Schoelkopf, P. Wahlgren, A. A. Kozhevnikov, P. Delsing, and D. E. Prober, "The Radio-Frequency Single-Electron Transistor (RF-SET): A Fast and Ultrasensitive Electrometer,” Science 280, 1238 (1998).

[Sillanpaa2004] M. A. Sillanpää, L. Roschier, and P. J. Hakonen, “Inductive Single-Electron Transistor,” Phys. Rev. Lett. 93, 066805 (2004).

[Sillapaa2005] M. A. Sillanpää, Quantum Device Applications of Mesoscopic Superconductivity, Ph.D. thesis, Helsinki University of Technology, Espoo, Finland (2005).

[Teufel2009] J. D. Teufel, T. Donner, M. A. Castellanos-Beltran, J. W. Harlow, and K. W. 
Lehnert, "Nanomechanical Motion Measured with an Imprecision below that at the Standard Quantum Limit,” Nature Nanotech. 4, 820 (2009).

[Wallraff2004] A. Wallraff, D. I. Schuster, A. Blais, L. Frunzio, R.-S. Huang, J. Majer, S. Kumar, S. M. Girvin, and R. J. Schoelkopf, "Strong Coupling of a Single Photon to a Superconducting Qubit Using Circuit Quantum Electrodynamics,” Nature 431, 162 (2004).

[Xue2009] W. W. Xue, Z. Ji, F. Pan, J. Stettenheim, M. P. Blencowe, and A. J. Rimberg, "Measurement of Quantum Noise in a Single Electron Transistor near the Quantum Limit," Nature Phys. 5, 660 (2009). 


\subsection{LIST OF SYMBOLS, ABBREVIATIONS, AND ACRONYMS}

cCPT: Cavity-embedded Cooper-pair transistor

CPT: $\quad$ Cooper-pair transistor

QD: Quantum dot

HEMT: $\quad$ High electron mobility transistor

RF-QPC: Radio-frequency quantum point contact

RF-SET: Radio-frequency single-electron transistor

SLUG: $\quad$ Superconducting low-inductance undulating galvanometer 